Ephemeride für r $\mathbf{2}^{\text {h }} \mathrm{m}$. Z. Berlin.

\begin{tabular}{|c|c|c|c|}
\hline 1882 & $a \mathscr{b}$ & $\delta \mathscr{y}$ & $\mathrm{H}$ \\
\hline $\begin{aligned} \text { Oct. } & 14 \\
& 18\end{aligned}$ & $\begin{array}{l}\text { 10 } \\
\text { 10 } 10^{\mathrm{m}} 25^{\mathrm{s}} \\
\text { 10 } 156\end{array}$ & $\begin{array}{r}-12^{\circ} 59^{\prime} 8 \\
1436.6\end{array}$ & $\begin{array}{l}0.59 \\
0.48\end{array}$ \\
\hline 22 & 10 941 & $16 \quad 10.2$ & 0.40 \\
\hline 26 & 10 42 & $\times 741.6$ & 0.34 \\
\hline 30 & $95^{8} \quad 2$ & $\begin{array}{ll}\text { I } & 10.7\end{array}$ & 0.29 \\
\hline Nov. 3 & $\begin{array}{lll}9 & 5134\end{array}$ & -20 35.4 & 0.26 \\
\hline
\end{tabular}

Als Einheit der Helligkeit gilt die vom 6. October.

Für die Beobachtung vom II. Sept. in Rio de Janeiro geben diese Elemente

$$
\alpha=10^{\mathrm{h}} \mathrm{I}^{\mathrm{m}} \quad \delta=-0^{\circ} 35^{\circ}
$$

während telegraphisch als Position des Cometen mitgetheilt wurde

$$
\alpha=9^{\mathrm{h}} 48^{\mathrm{m}} \quad \delta=-\mathrm{z}^{\circ} \mathrm{I}^{\prime}
$$

und es ist deshalb erklärlich, dass die erste in Wien mit Zugrundelegung dieser Angabe versuchte Bahnberechnung zu keinem Ziele führen konnte, und dadurch die Ausgabe eines Cometencirculares bis zum 2. October sich verzögerte.

\section{Währing 1882 October I 5 .}

Karl Zelbr,

Assistent der K. K. Sternwarte zu Wien.

\title{
Osservazione della cometa Cruls
}

\begin{tabular}{|c|c|c|c|c|c|c|c|}
\hline 1882 & T. M. Pal. & $\Delta \alpha$ & $\Delta \delta$ & Confr. & $\alpha$ app. & $\delta$ app. & $*$ \\
\hline Ott. 6 & $16^{\mathrm{h}} 59^{\mathrm{m}} 33^{\mathrm{s}}$ & $-\mathrm{I}^{\mathrm{m}} 39^{\mathrm{s}} 8 \mathrm{8}$ & $-8^{\prime}{ }_{4} \mathrm{r}^{\prime \prime} 8$ & 6 & $10^{\mathrm{h}} 3 \mathrm{r}^{\mathrm{m}} 24^{\mathrm{s}} \cdot 7^{6}$ & $-9^{\circ} 40^{\prime} 55^{\prime \prime} 4$ & $\mathbf{I}$ \\
\hline
\end{tabular}

fatta dal Direttore Cacciatore al micrometro anulare del Refrattore di o. $_{25}$ del R. Osservatorio di Palermo.

\begin{tabular}{|c|c|c|c|}
\hline * & $\alpha 1882.0$ & $\delta \times 882.0$ & A utorit à \\
\hline $\mathbf{I}$ & $10^{\mathrm{h}} 33^{\mathrm{m}} 2^{\mathrm{s}} \cdot 54$ & $-9^{\circ} 32^{\prime} \quad 2^{\prime \prime} \cdot 2$ & ${ }_{7} 6_{5}$ Lamont \\
\hline
\end{tabular}

Stella di confronto.

Il nucleo della cometa che la mattina del 5 presentavasi sotto forma oblunga avente un punto assai lucido nella parte inferiore, ed un altro meno lucido nella superiore, circondati ambedue i punti da dense nebulosità divise da un tratto oscuro, questa mattina si è mostrato più raccorciato, i punti lucidi sono meno vivaci, ed è circondato da più forte nebulosità.

Palermo I 882 Ott. 7 .

Il Direttore G. Cacciatore.

Osservazioni della cometa Cruls

\begin{tabular}{|c|c|c|c|c|c|c|c|c|c|}
\hline I $88 \mathrm{I}$ & T. M. Roma & $\Delta \alpha$ & $\Delta \delta$ & Confr. & $\alpha$ app. & $\log p .4$ & $\delta$ app. & $\log p .4$ & $*$ \\
\hline $\begin{array}{lr}\text { Ott. } & 6 \\
& \text { I I }\end{array}$ & $\begin{array}{lll}17^{\mathrm{h}} & 2^{\mathrm{m}} & 1^{\mathrm{s}} \\
\mathrm{I} & 24 & 9\end{array}$ & $+\bar{o}^{\mathrm{m}} 55^{5} \cdot 36$ & $-0^{\prime} 8^{\prime \prime \prime}$ & $\overline{5.5}$ & $\begin{array}{l}10^{\mathrm{h}} 31^{\mathrm{m}_{2}} 5^{\mathrm{s}} .92 \\
1024 \\
10.97\end{array}$ & $\overline{9.564 n}$ & $\begin{array}{rrr}-9^{\circ} & 40^{\prime} & 57^{\prime \prime} \\
-11 & 51 & 3.9\end{array}$ & $\overline{0.817}$ & \\
\hline
\end{tabular}

fatte all' equatoriale del R. Osservatorio del Collegio Romano.

Ste lla d i c on f r o n t o.

\begin{tabular}{c|c|c|c}
\hline$*$ & $\alpha 1882.0$ & $\delta 1882.0$ & A u t o r i ta \\
\hline Ott. I I & $10^{\mathrm{h}} 23^{\mathrm{m}} 188_{39}+2^{\mathrm{s}} 22$ & $-\mathrm{Ir}^{\circ} 49^{\prime} 54^{\prime \prime} 9-10^{\prime \prime} 9$ & Trettenero Santini Cat. di 2696 St. Nr. 1264.
\end{tabular}

E. Millosevich. 